\title{
Supersymmetry of Noncompact MQCD-like Membrane Instantons and Heat Kernel Asymptotics
}

\author{
Kanishka Belani ${ }^{1}$, Payal Kaura ${ }^{2}$ and Aalok Misra ${ }^{3}$ \\ Indian Institute of Technology Roorkee, \\ Roorkee - 247 667, Uttaranchal, India
}

\begin{abstract}
We perform a heat kernel asymptotics analysis of the nonperturbative superpotential obtained from 'wrapping of an $M 2$-brane around a supersymmetric noncompact three-fold embedded in a (noncompact) ' $G_{2}$-manifold as obtained in [1], the three-fold being the one relevant to domain walls in Witten's MQCD '[2], in the limit of small " $\zeta$ ", a complex constant that appears in the Riemann surfaces relevant to defining the boundary conditions for the domain wall in MQCD. The MQCD-like configuration is interpretable, "for small but non-zero $\zeta$ as a noncompact/"large" open membrane instanton, and for vanishing $\zeta$, as the type IIA D0-brane (for vanishing $M$-theory circle radius). We find that the eta-function Seeley de-Witt coefficients vanish, and we get a perfect match between the zeta-function Seeley de-Witt coefficients (up to terms quadratic in $\zeta$ ) between the Dirac-type operator and one of the two Laplace-type operators 'figuring in the superpotential. Given the dissimilar forms of the bosonic and the square of the fermionic operators, this is an extremely nontrivial check, from a spectral analysis point of view, of the expected residual supersymmetry for the nonperturbative configurations in $M$-theory considered in this work.
\end{abstract}

\footnotetext{
${ }^{1}$ email: imkani@hotmail.com

${ }^{2}$ email: pa123dph@iitr.ernet.in

3 e-mail: aalokfph@iitr.ernet.in
} 


\section{Introduction}

String and $M$ theories on manifolds with $G_{2}$ and $\operatorname{Spin}(7)$ holonomies have become an active area of research, after construction of explicit examples of such manifolds by Joyce[3]. Some explicit metrics of noncompact manifolds with the above-mentioned exceptional holonomy groups have been constructed by Brandhuber et al and Cvectic et al [4].

Further, it will be interesting to be able to lift the Gopakumar-Vafa Chern-Simons/closed type Atopological open/closed string duality to $M$ theory on a $G_{2}$-holonomy manifold, without having to embed it first into type IIA string theory as was done by Vafa. As the type-A topological string theory's partition function receives contributions only from holomorphic maps from the world-sheet to the target space, and apart from constant maps, instantons fit the bill, as a first step we should look at obtaining the superpotential contribution of wrapping of an $M 2$ brane on supersymmetric 3-cycle in a suitable $G_{2}$-holonomy manifold(membrane instantons). In terms of relating the result obtained in [5](on membrane instanton superpotential in terms of bosonic and fermionic determinants) to that of the 1-loop Schwinger computation of $M$ theory and the large $N$-limit of the partition function evaluated in [6], one notes that the 1-loop Schwinger computation also has as its starting point, an infinite dimensional bosonic determinant of the type $\operatorname{det}\left((i \partial-e A)^{2}-Z^{2}\right), A$ being the gauge field corresponding to an external self-dual field strength, and $Z$ denoting the central charge. The large $N$-limit of the partition function of Chern Simons theory on an $S^{3}$, as first given by Periwal in [7], involves the product of infinite number of sin's, that can be treated as the eigenvalues of an infinite dimensional determinant. This is indicative of a possible connection between the membrane instanton contribution to the superpotential, the 1-loop Schwinger computation and the large $N$ limit of the Chern-Simons theory on an $S^{3}$ (See also [8]).

After the construction of noncompact (special Lagrangian) three-folds of Joyce [9] given by the following equations (noncompact SLAGS in $\mathbf{C}^{3}$ ): $\left|z_{i}\right|^{2}-t=\left|z_{j}\right|^{2}=\left|z_{k}\right|^{2}, \operatorname{Re}\left(z_{1} z_{2} z_{3}\right) \geq 0, \operatorname{Im}\left(z_{1} z_{2} z_{3}\right)=0, t>0, i \neq$ $j \neq k=1,2,3$, the same have been studied in the context of wrapping of $D 6$-branes around noncompact SLAGS diffeomorphic to $S^{1} \times \mathbf{R}^{2}$ in [10] - see also [11]. In this paper, we identify a noncompact instantonic configuration in M-theory compactified on a $G_{2}$-manifold with a particular supersymmetric noncompact three-fold embedded in the same, as relevant to domain walls in MQCD. What this means is that we consider $M$ theory compactified on the same $G_{2}$-manifold with a supersymmetric noncompact three-fold embedded in it that appears in the study of domain walls in MQCD, but we do not work with MQCD - we just "borrow" the MQCD-domain-wall $G_{2}$, but continue working with $M$ theory which gives $\mathcal{N}=1, D=4$ chiral multiplets (in addition to gravity and $U(1)$ vector multiplets) - there are no chiral multiplets in $\mathcal{N}=1$ MQCD. We are considering the superpotential of an isolated membrane instanton obtained by wrapping of an M2-brane on the aforementioned supersymmetric three-fold. We provide evidence for the expected residual supersymmetry for the same, from a spectral analysis point of view, by looking at the Seeley-de Witt coefficients associated with the Laplace-type and Dirac-type operators relevant to the nonperturbative superpotential for small value of a complex constant " $\zeta$ " that figures in Riemann surfaces relevant to MQCD. Notice that the reason why from the heat kernel asymptotics' point of view, the fact that the instantonic configuration possesses some surviving supersymmetry is not obvious is because if one looks at the forms of the Laplace-type (bosonic) operator (the relevant one denoted in this paper by $\mathcal{O}_{1}$ ) and the Dirac-type (fermionic) operator (denoted in this paper by $\mathcal{O}_{3}$ ), then writing $\mathcal{O}_{1}=G^{i j} \partial_{i} \partial_{j}+X_{1}$ and $\mathcal{O}_{3}^{2}=G^{i j} \partial_{i} \partial_{j}+X_{2}$, one sees that $X_{1}$ is not the same as $X_{2}$. Had $X_{1}$ equalled $X_{2}$, then using a theorem of McKean and Singer ([12]), one would have been assured of absence of UV divergence in $\ln \operatorname{det} \mathcal{O}_{1}-\ln \operatorname{det} \mathcal{O}_{3}$. The fact that, as 
we will show in section 4 , one still gets a perfect cancelation (to the order considered in our work), is, we feel, a very non-trivial result.

The main idea behind this paper is to use the heat kernel asymptotics techniques to see whether or not the aforementioned membrane instanton superpotential receives quantum corrections from the bosonic and fermionic fluctuations, thereby verifying the expected (because one is using a supersymmetric three-fold for wrapping the M2-brane) surviving supersymmetry. Further, as far as we know, a spectral analysis for supersymmetric three-folds embedded in a $G_{2}$-manifold, has never been done earlier.

The plan of the paper is as follows. In section $\mathbf{2}$, we review the calculation of the membrane instanton superpotential of [5], which is based on [13] - for this work, given the non-singular uplift to M-theory in MQCD, we assume that there is no contribution to the superpotential from the membrane boundary. In section 3, we discuss spin connections for associative three-cycles based on the discussion on the same in [14]. In section 4, we perform a heat kernel asymptotics analysis for the superpotential of section $\mathbf{2}$ and using the results of [15], evaluate the bulk and boundary Seeley de-Witt coefficients for one of the two Laplacetype operators and the Dirac-type operator. We obtain a remarkably perfect match between the two (up to $\mathcal{O}\left(\zeta^{2}\right)$ ) thereby strongly indicative of surviving supersymmetry of the nonperturbative configurations in $M$-theory considered in this work.

\section{Evaluation of the membrane instanton contribution to the superpo- tential}

In [5], one of us (AM) had worked out the membrane instanton superpotential, using techniques developed in [13], based on the path-integral-inside-a-path integral approach of [18]. We briefly review the same first.

As given in [14], the Euclidean action for an $M 2$ brane is given by the following Bergshoeff, Sezgin, Townsend action:

$$
\mathcal{S}_{\Sigma}=\int_{\Sigma} d^{3} z\left[\frac{\sqrt{g}}{l_{11}^{3}}-\frac{i}{3 !} \epsilon^{i j k} \partial_{i} \mathbf{Z}^{M} \partial_{j} \mathbf{Z}^{N} \partial_{k} \mathbf{Z}^{P} C_{M N P}(X(s), \Theta(s))\right],
$$

where $\mathbf{Z}$ is the map of the $M 2$ brane world-volume to the the $D=11$ target space $M_{11}$, both being regarded as supermanifolds and $\Sigma$ is the $M 2$-brane world volume. The $g$ in (1), is defined as:

$$
g_{i j}=\partial_{i} \mathbf{Z}^{M} \partial_{j} \mathbf{Z}^{N} \mathbf{E}_{M}^{A} \mathbf{E}_{N}^{B} \eta_{A B},
$$

where $\mathbf{E}_{M}^{A}$ is the supervielbein, given in [14]. $X(s)$ and $\Theta(s)$ are the bosonic and fermionic coordinates of Z. After using the static gauge and $\kappa$-symmetry fixing, the physical degrees of freedom, are given by $y^{m^{\prime \prime}}$, the section of the normal bundle to the $M 2$-brane world volume, and $\Theta(s)$, section of the spinor bundle tensor product: $S(T \Sigma) \otimes S^{-}(N)$, where the - is the negative $S p i n(8)$ chirality, as under an orthogonal decomposition of $\left.T M_{11}\right|_{\Sigma}$ in terms of tangent and normal bundles, the structure group Spin(11) decomposes into $\operatorname{Spin}(3) \times \operatorname{Spin}(8)$.

The action in (1) needs to be expanded up to $O\left(\Theta^{2}\right)$, and the expression is (one has to be careful that in Euclidean $D=11$, one does not have a Majorana-Weyl spinor or a Majorana spinor) given as:

$$
\begin{aligned}
& \mathcal{S}_{\Sigma}=\int_{\Sigma}\left[C+\frac{i}{l_{11}^{3}} \operatorname{vol}(g)+\frac{\sqrt{g}}{l_{11}^{3}}\left(g^{i j} D_{i} y^{m^{\prime \prime}} D_{j} y^{n^{\prime \prime}} h_{m^{\prime \prime} n^{\prime \prime}}-y^{m^{\prime \prime}} \mathcal{U}_{m^{\prime \prime} n^{\prime \prime}} y^{n^{\prime \prime}}+O\left(y^{3}\right)\right)\right. \\
& \left.+\frac{i}{l_{11}^{3}} \sqrt{g} \frac{1}{2}\left(\bar{\Psi}_{M} V^{M}-\bar{V}^{M} \Psi_{M}\right)+2 \frac{\sqrt{g}}{l_{11}^{3}} g^{i j} \bar{\Theta} \Gamma_{i} D_{j} \Theta+O\left(\Theta^{3}\right)\right]
\end{aligned}
$$


where we follow the conventions of [14]: $V_{M}$ being the gravitino vertex operator, $\Psi$ being the gravitino field that enters via the supervielbein $\mathbf{E}_{M}^{A}, \mathcal{U}$ is a mass matrix defined in terms of the Riemann curvature tensor and the second fundamental form, $C$ is the pull-back of the $M$-theory three-form potential on to the world volume of the $M 2$-brane and $\Gamma_{i}$ s are pull-backs of the eleven-dimensional gamma matrices on to $\Sigma$.

After $\kappa$-symmetry fixing, like [14], we set $\Theta_{2}^{A \dot{a}}(s)(A$ and $\dot{a}$ index the $\operatorname{Spin}(3)$ and the positive-chirality $\operatorname{Spin}(8)$ groups respectively), i.e., the positive $\operatorname{Spin}(8)$-chirality, to zero, and following [13], will refer to $\Theta_{1}^{A a}(s)$ as $\theta$.

The Kaluza-Klein reduction of the $D=11$ gravitino $\Psi_{M}$, is given by: $d x^{M} \Psi_{M}=d x^{\mu} \Psi_{\mu}+d x^{m} \Psi_{\mu},(\mu$ indexes the four-dimensional Euclidean space $\mathbf{R}^{4}(x)$ and $m$ indexes the $G_{2}$ seven-fold $\left.X_{G_{2}}(y)\right) \Psi_{\mu}(x, y)=$ $\psi_{\mu}(x) \otimes \vartheta(y), \Psi_{m}(x, y)=l_{11}^{3} \sum_{i=1}^{b_{3}} \omega_{i, m n p}^{(3)}(y) \Gamma^{p q} \chi^{i}(x) \otimes \eta(y), \omega^{(3)} \in H^{3}\left(X_{G_{2}}\right)$, where we do not write the terms obtained by expanding in terms of a basis of the harmonic 2-forms of $H^{2}\left(X_{G_{2}}\right)$, as we will be interested in $M 2$ branes wrapping supersymmetric 3-cycles in the $G_{2}$-holonomy manifold - $\Gamma^{p q}$ is the antisymmetrized product of two $C l(0,11)$ generators, $\psi_{\mu}$ is the four-dimensional gravitino in the four-dimensional $\mathcal{N}=1$ (super)gravity multiplet, $\chi^{i}$ are the four-dimensional fermions in the $\mathcal{N}=1$ chiral multiplet ( $M$-theory compacitified on a $G_{2}$ manifold would yield a four-dimensional $\mathcal{N}=1$ theory) and $\eta(y)$ is a covariantly constant spinor on the $G_{2}$-manifold. For evaluating the nonperturbative contribution to the superpotential, following [14], we will evaluate the fermionic 2-point function: $\left\langle\chi^{i}\left(x_{1}^{u}\right) \chi^{j}\left(x_{2}^{u}\right)\right\rangle$ (where $x_{1,2}$ are the $\mathbf{R}^{4}$ coordinates and $\mathrm{u}$ [and later also $v$ ] $\equiv 7,8,9,10$ is [are] used to index these coordinates), and drop the interaction terms in the $D=4, \mathcal{N}=1$ supergravity action. The corresponding mass term in the supergravity action appears as $\partial_{i} \partial_{j} W$, where the derivatives are evaluated w.r.t. the complex scalars obtained by the Kaluza-Klein reduction of $C+\frac{i}{l_{11}^{3}} \Phi$ ( $\Phi$ being the closed as well as co-closed $G_{2}$-calibration three form defined over $X_{G_{2}}$ ) using harmonic three forms forming a basis for $H^{3}\left(X_{G_{2}}, \mathbf{R}\right)$. One then integrates twice to get the expression for the superpotential from the 2-point function.

The bosonic zero modes are the four bosonic coordinates that specify the position of the supersymmetric 3 -cycle, and will be denoted by $x_{0}^{7,8,9,10} \equiv x_{0}^{u}$. The fermionic zero modes come from the fact that for every $\theta_{0}$ that is the solution to the fermionic equation of motion, one can always shift $\theta_{0}$ to $\theta_{0}+\theta^{\prime}$, where $D_{i} \theta^{\prime}=0$. This $\theta^{\prime}=\vartheta \otimes \eta$ where $\vartheta$ is a $D=4$ Weyl spinor, and $\eta$ is a covariantly constant spinor on the $G_{2}$-holonomy manifold.

After expanding the $M 2$-brane action in fluctuations about solutions to the bosonic and fermionic equations of motion, one gets that: $\left.\mathcal{S}\right|_{\Sigma}=\mathcal{S}_{0}^{y}+\mathcal{S}_{0}^{\theta}+\mathcal{S}_{2}^{y}+\mathcal{S}_{2}^{\theta}$, where $\left.\mathcal{S}_{0}^{y} \equiv \mathcal{S}_{\Sigma}\right|_{y_{0}, \theta_{0}} \mathcal{S}_{0}^{\theta} \equiv \mathcal{S}_{\Sigma}^{\theta}+\left.\mathcal{S}_{\Sigma}^{\theta^{2}}\right|_{y_{0}, \theta_{0}}$; $\left.\mathcal{S}_{2}^{y} \equiv \frac{\delta^{2} \mathcal{S}_{\Sigma}}{\delta y^{2}}\right|_{y_{0}, \theta_{0}=0}(\delta y)^{2} ;\left.\mathcal{S}_{2}^{\theta} \equiv \frac{\delta^{2} \mathcal{S}}{\delta \theta^{2}}\right|_{y_{0}, \theta_{0}=0}(\delta \theta)^{2}$. Following [13], we consider classical values of coefficients of $(\delta y)^{2},(\delta \theta)^{2}$ terms, as fluctuations are considered to be of $\mathcal{O}\left(\sqrt{\alpha^{\prime}}\right)$.

Now,

$$
\begin{aligned}
& \left\langle\chi^{i}\left(x_{1}^{u}\right) \chi^{j}\left(x_{2}^{u}\right)\right\rangle= \\
& \int \mathcal{D} \chi e^{K . E} \text { of } \chi \chi^{i}(x) \chi^{j}(x) \int d^{4} x_{0} e^{-\mathcal{S}_{0}^{y}} \\
& \times \int d \vartheta^{1} d \vartheta^{2} e^{-\mathcal{S}_{0}^{\theta}} \int \mathcal{D} \delta y^{m^{\prime \prime}} e^{-\mathcal{S}_{2}^{y}} \int \mathcal{D} \delta \bar{\theta} \mathcal{D} \delta \theta e^{-\mathcal{S}_{2}^{\theta}} .
\end{aligned}
$$

We now evaluate the various integrals that appear in (4) above starting with $\int d^{4} x e^{-\mathcal{S}_{0}^{y}}$ :

$$
\int d^{4} x_{0} e^{-\mathcal{S}_{0}^{y}}=\int d^{4} x_{0} e^{\left[i C-\frac{1}{l_{11}^{3}} \operatorname{vol}(g)\right]} .
$$


Using the 11-dimensional Euclidean representation of the gamma matrices as given in [14], $\mathcal{S}_{0}^{\theta}+\left.\mathcal{S}_{0}^{\theta^{2}}\right|_{\Sigma}=$ $\frac{i}{2 l_{11}^{3}} \int_{\Sigma} \sqrt{g} \bar{\Psi}_{M} V_{M} d^{3} s$, where using $\partial_{i} x_{0}^{u}=0$, and using $U$ to denote coordinates on the $G_{2}$-holonomy manifold, $V^{U}=g_{i j} \partial_{i} y_{0}^{U} \partial_{j} y^{V} \gamma_{V} \theta_{0}+\frac{i}{2} \epsilon^{i j k} \partial_{i} y_{0}^{U} \partial_{j} y_{0}^{V} \partial_{k} y_{0}^{W} \Gamma_{V W} \theta_{0}$,

$$
\int d \vartheta_{1} d \vartheta_{2} e^{\frac{i}{2 l_{11}^{3}} \sum_{I=1}^{b_{3}} \sum_{\alpha=1}^{2} \sum_{i=1}^{8}\left(\bar{\chi}(x) \sigma^{(i)}\right)_{\alpha} \vartheta_{\alpha} \omega_{I}^{(i)}}=-\frac{1}{4 l_{11}^{3}} \sum_{I=1}^{b_{3}} \sum_{i<j=1}^{8} \omega_{I}^{(i)} \omega_{I}^{(j)}\left(\bar{\chi} \sigma^{(i)}\right)_{1}\left(\bar{\chi} \sigma^{(j)}\right)_{2},
$$

where one uses that for $G_{2}$-spinors, the only non-zero bilinears are: $\eta^{\dagger} \Gamma_{i_{1} \ldots i_{p}} \eta$ for $p=0$ (三 constant), $p=3(\equiv$ calibration 3-form), $p=4$ (三 Hodge dual of the calibration 3-form) and $p=7$ (三 volume form). We follow the following notations for coordinates: $u, v$ are $\mathbf{R}^{4}$ coordinates and $U, V$ index $G_{2}$-holonomy manifold coordinates. The tangent/curved space coordinates for $\Sigma$ are represented by $a^{\prime} / m^{\prime}$ and those for $X_{G_{2}} \times \mathbf{R}^{4}$ are represented by $a^{\prime \prime} / m^{\prime \prime}$.

We now come to the evaluation of $\left.\mathcal{S}_{2}^{\theta}\right|_{y_{0}, \theta_{0}=0}$. Using the equality of the two $O\left((\delta \Theta)^{2}\right)$ terms in the action of Harvey and Moore, and arguments similar to the ones in [13], one can show that one needs to evaluate the following bilinears: $\delta \bar{\Theta} \Gamma_{a^{\prime}} \partial_{i} \delta \Theta, \delta \bar{\Theta} \Gamma_{a^{\prime \prime}} \partial_{i} \delta \Theta, \delta \bar{\Theta} \Gamma_{a^{\prime}} \Gamma_{A B} \delta \Theta$, and $\delta \bar{\Theta} \Gamma_{m^{\prime \prime}} \Gamma_{A B} \delta \Theta$. Evaluating them, one gets:

$$
\left.\mathcal{S}_{2}^{\theta}\right|_{y_{0}, \theta_{0}=0} \equiv \int_{\Sigma} d^{3} s \delta \theta^{\dagger} \mathcal{O}_{3} \delta \theta
$$

where $\mathcal{O}_{3} \equiv \sqrt{g} g^{i j} \Gamma_{i} D_{j}$, the precise definition of $\Gamma_{i}$ will be given later. Hence, the integral over the fluctuations in $\theta$ will give a factor of $\sqrt{\operatorname{det} \mathcal{O}_{3}}$ in Euclidean space.

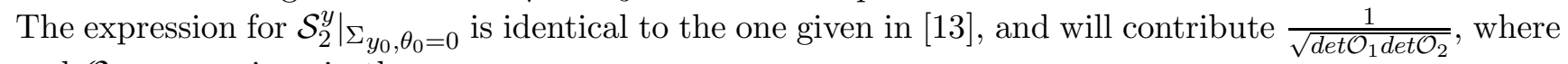
$\mathcal{O}_{1}$ and $\mathcal{O}_{2}$ are as given in the same paper:

$$
\begin{aligned}
& \mathcal{O}_{1} \equiv \eta_{u v} \sqrt{g} g^{i j} \mathcal{D}_{i} \partial_{j} \\
& \mathcal{O}_{2} \equiv \sqrt{g}\left(g^{i j} \mathcal{D}_{i} h_{\hat{U} \hat{V}} D_{j}+\mathcal{U}_{\hat{U} \hat{V}}\right) .
\end{aligned}
$$

The mass matrix $\mathcal{U}$ is expressed in terms of the curvature tensor and product of two second fundamental forms. $\mathcal{D}_{i}$ is a covariant derivative with indices in the corresponding spin-connection of the type $\left(\omega_{i}\right)_{n^{\prime \prime}}^{m^{\prime \prime}}$ and $\left(\omega_{i}\right)_{n^{\prime}}^{m^{\prime}}$, and $D_{i}$ is a covariant derivative with corresponding spin connection indices of only the latter type.

Hence, modulo supergravity determinants, and the contribution from the fermionic zero modes, the exact form of the superpotential contribution coming from a single $M 2$ brane wrapping an isolated supersymmetric cycle of $G_{2}$-holonomy manifold, is given by:

$$
\Delta W=e^{i C-\frac{1}{l_{11}^{3}} \operatorname{vol}(h)} \sqrt{\frac{\operatorname{det} \mathcal{O}_{3}}{\operatorname{det} \mathcal{O}_{1} \operatorname{det} \mathcal{O}_{2}}} .
$$

Note that the result (9), unlike that of [14], is also applicable for non-rigid three-cycles (implying $b_{1}(\Sigma) \neq 0$ ). We do not bother about 5-brane instantons, as we assume that $H_{6}=0$ for the $G_{2}$-manifold. One should bear in mind that it is only for compact $G_{2}$-manifolds $X_{7}$ that $H_{7}^{4}\left(X_{7}\right)$, valued in the seven-dimensional representation of the $G_{2}$-group, and therefore $H_{3}\left(X_{7}\right)$ vanishes - note however $H^{4}\left(X_{7}\right)=H_{1}^{4}\left(X_{7}\right) \oplus H_{7}^{4}\left(X_{7}\right) \oplus$ $H_{27}^{4}\left(X_{7}\right)$ (for a compact $X_{7}$ ) - hence (for a compact $X_{7}$ ), $H^{4}\left(X_{7}\right)$ and hence $H_{3}\left(X_{7}\right)$ is non-trivial. Besides, we are working with a noncompact $G_{2}$ manifold [16]. Even though we have turned off the $G$-flux and the calibration three-form characterizing $X_{7}$ is closed, $H^{4}\left(X_{7}\right)$ and therefore $H_{3}\left(X_{7}\right)$ are still non-trivial. Part of the reason is the shift of the quantization of the $G$-flux (See $[17]):\left[\frac{G}{2 \pi}\right]-\frac{\lambda}{2} \in H^{4}\left(X_{7}, \mathbf{Z}\right.$ ), where 
the characteristic class $\lambda$ is given by $\frac{p_{1}\left(X_{7}\right)}{2}, p_{1}\left(X_{7}\right)$ being the first Pontryagin class. For a $G_{2}$ manifold, $p_{1}\left(X_{7}\right)=p_{1}\left(X_{8}=X_{7} \times S^{1}\right), X_{7} \times S^{1}$ being a spin eight-manifold (See [14]), for which $p_{1}$ is even. According to the Wu's formula (See [17]), the intersection form of a spin eight-fold satisfies the following relation: $x^{2} \cong x \wedge \lambda \bmod 2$, where $x \in H^{4}\left(X_{8}, \mathbf{Z}\right)$. Hence, for a spin manifold, $\lambda$ is even if the intersection form is even - the intersection form for $X_{8}$ is even, thereby justifying the switching off of $G$. Further, $p_{1}\left(X_{7}\right) \neq 0^{4}$ implying that $H^{4}\left(X_{7}\right)$ and therefore $H_{3}\left(X_{7}\right)$ can not be trivial.

To actually evaluate the Seeley-de Witt coefficients for Laplace-type operators (See (34)) we need to find an example of a regular $G_{2}$-holonomy manifold that is locally $\Sigma \times M_{4}$, where $\Sigma$ is a supersymmetric 3 -cycle on which we wrap an $M 2$ brane once, and $M_{4}$ is a four manifold. The condition for supersymmetric cycle: $\left.\Phi\right|_{\Sigma}=\operatorname{vol}(\Sigma)$, is what is solved for in [1]. Such a three-fold will be discussed in section 4 in the context of MQCD.

\section{Spin Connection for Associative 3-Cycles in $G_{2}$ Manifolds}

We now discuss how to figure out the independent components of the spin connection of the type $\omega_{i}^{a^{\prime} b^{\prime \prime}}$ (the superindices indexing the tangent space indices as explained below equation (6) above: $i$ indexes $\Sigma, a^{\prime}$ indexes the tangent space of $\Sigma$ and $b^{\prime \prime}$ indexes the tangent space corresponding to directions normal to $\Sigma$ ).

The bi-spinorial representation of the components of the spin connection $\omega_{i}^{a^{\prime} b^{\prime}}, \omega_{i}^{a^{\prime \prime}} b^{\prime \prime}, \omega_{i}^{a^{\prime} b^{\prime \prime}}$ can be worked out as below. Let $\omega_{i}^{a^{\prime} b^{\prime}} \equiv \epsilon^{a^{\prime} b^{\prime} c^{\prime}} \omega_{i}^{c^{\prime}}$. We can then construct $\omega_{i}^{A B} \equiv\left(\sum_{a^{\prime}=1}^{3} \omega_{i}^{a^{\prime}+2} \sigma^{a^{\prime}}\right) A B \equiv \omega_{i}^{A B}$ (where $\sigma^{a^{\prime}}$ are the Pauli matrices and $A, B$ are the bispinorial indices), abbreviated as $\omega_{\|}$. The components $\omega_{i}^{a^{\prime \prime} b^{\prime \prime}}$, with $a^{\prime \prime}, b^{\prime \prime}=6,7,8,10$ can be split into three self-dual and three anti-self-dual components:

$$
\begin{aligned}
& \omega_{i}^{67}+\omega_{i}^{810} \equiv\left(\omega_{i}^{+}\right)^{1}, \\
& \omega_{i}^{68}+\omega_{i}^{107} \equiv\left(\omega_{i}^{+}\right)^{2}, \\
& \omega_{i}^{610}+\omega_{i}^{78} \equiv\left(\omega_{i}^{+}\right)^{3},
\end{aligned}
$$

and

$$
\begin{aligned}
& \omega_{i}^{67}-\omega_{i}^{810} \equiv\left(\omega_{i}^{-}\right)^{1}, \\
& \omega_{i}^{68}-\omega_{i}^{107} \equiv\left(\omega_{i}^{-}\right)^{2}, \\
& \omega_{i}^{610}-\omega_{i}^{78} \equiv\left(\omega_{i}^{-}\right)^{3},
\end{aligned}
$$

from which one constructs $\sum_{a=1}^{3}\left(\omega_{i}^{+}\right)^{a} \sigma^{a} \equiv\left(\omega_{i}^{+}\right)^{Y Y^{\prime}}$, abbreviated as $\omega_{\perp}^{+}$, and $\sum_{a=1}^{3}\left(\omega_{i}^{-}\right)^{a} \sigma^{a} \equiv\left(\omega_{i}^{-}\right)^{\dot{Y} \dot{Y}^{\prime}}$, abbreviated as $\omega_{\perp}^{-}$. For the "off-diagonal" components $\omega^{a^{\prime} b^{\prime \prime}}$, one constructs $\sum_{a^{\prime}=1}^{3}\left(\omega^{a^{\prime} b^{\prime \prime}}\right) \sigma^{a^{\prime}} \equiv\left(\omega_{i}\right)^{A B b^{\prime \prime}}$, and further $\left(\omega_{i}^{A B 6} \mathbf{1}_{2}+\omega_{i}^{A B 7} \sigma^{1}+\omega_{i}^{A B 8} \sigma^{2}+\omega_{i}^{A B} 10 \sigma^{3}\right)^{Y \dot{Y}} \equiv(\omega)_{i}^{A B Y \dot{Y}}$.

For associative three-cycles, $(\omega)_{i}^{A B Y Y}$ is symmetric w.r.t. $A, B$ and $Y$ and $\omega_{\|}=\omega_{\perp}^{-}$(See [14].). Assuming $\omega_{\perp}^{+}=0$, implying $\omega_{i}^{67}=-\omega_{i}^{810}, \omega_{i}^{68}=-\omega_{i}^{107}, \omega_{i}^{6} 10=-\omega_{i}^{78}$, the relation $\omega_{\|}=\omega_{\perp}^{-}$implies: $\frac{1}{2} \omega_{i}^{1}=$

\footnotetext{
${ }^{4}$ As an example of a compact $X_{7}$, one could consider $\frac{T^{7}}{\Gamma}$ with fixed points for $\Gamma$ - see [3] - $p_{1}\left(X_{7}\right)$ for compact $X_{7}$ with holonomy given by $G_{2}$, is non-zero and satisfies the equation: $\left\langle p_{1} \cup \phi,\left[X_{7}\right]\right\rangle=-\frac{1}{8 \pi^{2}} \int_{X_{7}}|R|^{2}<0$ (See [16]), $\phi$ being the (co)closed calibration three-form
} 
$\omega_{i}^{67}, \frac{1}{2} \omega_{i}^{2}=\omega_{i}^{68}, \frac{1}{2} \omega_{i}^{3}=\omega_{i}^{6} 10$. Hence,

$$
\omega_{i}^{a^{\prime \prime} b^{\prime \prime}}=\left(\begin{array}{cccc}
0 & \frac{1}{2} \omega_{i}^{1} & \frac{1}{2} \omega_{i}^{2} & \frac{1}{2} \omega^{3} \\
-\frac{1}{2} \omega_{i}^{1} & 0 & -\frac{1}{2} \omega_{i}^{3} & \frac{1}{2} \omega_{i}^{2} \\
-\frac{1}{2} \omega_{i}^{1} & \frac{1}{2} \omega_{i}^{3} & 0 & -\frac{1}{2} \omega_{i}^{61} \\
-\frac{1}{2} \omega_{i}^{3} & -\frac{1}{2} \omega_{i}^{2} & -\frac{1}{2} \omega_{i}^{61} & 0
\end{array}\right)
$$

Now,

$$
\left(\sum_{a^{\prime}=1}^{3} \omega_{i}^{a^{\prime}+2} b^{\prime \prime} \sigma^{a^{\prime}}\right)^{A B}=\left(\begin{array}{cc}
\omega_{i}^{5 b^{\prime \prime}} & \omega_{i}^{3 b^{\prime \prime}}-i \omega_{i}^{4 b^{\prime \prime}} \\
\omega_{i}^{3 b^{\prime \prime}}+i \omega_{i}^{4 b^{\prime \prime}} & -\omega_{i}^{5 b^{\prime \prime}}
\end{array}\right)^{A B}
$$

Hence, for $A=B=1$ or 2 , consider

$$
\begin{aligned}
& \pm\left(\omega_{i}^{56} \mathbf{1}_{2}+\omega_{i}^{57} \sigma^{1}+\omega_{i}^{58} \sigma^{2}+\omega_{i}^{510} \sigma^{3}\right)^{Y \dot{Y}} \\
& = \pm\left(\begin{array}{cc}
\omega_{i}^{56}+\omega_{i}^{510} & \omega_{i}^{57}-i \omega_{i}^{58} \\
\omega_{i}^{57}+i \omega_{i}^{58} & \omega_{i}^{56}-\omega_{i}^{5} 10
\end{array}\right)^{Y \dot{Y}}
\end{aligned}
$$

and for $A=1, B=2$, consider:

$$
\begin{aligned}
& \left(\left(\omega_{i}^{36}-i \omega_{i}^{46}\right) \mathbf{1}_{2}+\left(\omega_{i}^{37}-i \omega_{i}^{47}\right) \sigma^{1}+\left(\omega_{i}^{38}-i \omega_{i}^{48}\right) \sigma^{2}+\left(\omega_{i}^{3110}-i \omega_{i}^{4} 10\right) \sigma^{3}\right)^{Y \dot{Y}} \\
& =\left(\begin{array}{cc}
\left(\omega_{i}^{36}-i \omega_{i}^{46}\right)+\left(\omega_{i}^{310}-i \omega_{i}^{410}\right) & \left(\omega_{i}^{37}-i \omega_{i}^{47}\right)-i\left(\omega_{i}^{38}-i \omega_{i}^{48}\right) \\
\left(\omega_{i}^{37}-i \omega_{i}^{47}\right)+i\left(\omega_{i}^{38}-i \omega_{i}^{48}\right) & \left(\omega_{i}^{36}-i \omega_{i}^{46}\right)-\left(\omega_{i}^{310}-i \omega_{i}^{410}\right)
\end{array}\right)^{Y \dot{Y}}
\end{aligned}
$$

Now, $\left(\omega_{i}\right)^{111 \dot{1}},\left(\omega_{i}\right)^{111 \dot{2}},\left(\omega_{i}\right)^{222 \dot{1}},\left(\omega_{i}\right)^{222 \dot{2}}$ are already symmetric in $A, B, Y$. Now,

$$
\begin{aligned}
& \left(\omega_{i}\right)^{112 \dot{1}}=\omega_{i}^{57}+i \omega_{i}^{58}, \\
& \left(\omega_{i}\right)^{121 \dot{1}}=\left(\omega_{i}^{36}+\omega_{i}^{310}\right)-i\left(\omega_{i}^{46}+\omega_{i}^{410}\right), \\
& \left(\omega_{i}\right)^{211 \dot{1}}=\left(\omega_{i}^{36}+i \omega_{i}^{310}\right)+i\left(\omega_{i}^{46}+\omega_{i}^{410}\right) .
\end{aligned}
$$

Hence, $\omega_{i}^{112 i}=\omega_{i}^{1211}=\omega_{i}^{2111}$ implies

$$
\omega_{i}^{57}=\omega_{i}^{36}+\omega_{i}^{310} ; \omega_{i}^{58}=0 ; \omega_{i}^{46}=-\omega_{i}^{410} .
$$

Similarly,

$$
\begin{aligned}
& \left(\omega_{i}\right)^{112 \dot{2}}=\omega_{i}^{56}-\omega_{i}^{510} \\
& \left(\omega_{i}\right)^{121 \dot{2}}=\left(\omega_{i}^{37}-\omega_{i}^{48}\right)-i\left(\omega_{i}^{47}+\omega_{i}^{38}\right) \\
& \left(\omega_{i}\right)^{211 \dot{2}}=\left(\omega_{i}^{37}+\omega_{i}^{48}\right)-i\left(-\omega_{i}^{47}+\omega_{i}^{38}\right)
\end{aligned}
$$

and their equality would imply:

$$
\omega_{i}^{47}=\omega_{i}^{38}=\omega_{i}^{48}=0 ; \omega_{i}^{37}=\omega_{i}^{56}-\omega_{i}^{510},
$$


and the equality of:

$$
\begin{aligned}
& \left(\omega_{i}\right)^{122 \dot{1}}=\left(\omega_{i}^{37}+\omega_{i}^{48}\right)+i\left(\omega_{i}^{38}-\omega_{i}^{47}\right), \\
& \left(\omega_{i}\right)^{212 \dot{1}}=\left(\omega_{i}^{37}-\omega_{i}^{48}\right)+i\left(\omega_{i}^{47}+\omega_{i}^{38}\right), \\
& \omega_{i}^{221 \dot{1}}=\omega_{i}^{510}-\omega_{i}^{56}
\end{aligned}
$$

implies

$$
\omega_{i}^{37}=\omega_{i}^{56}=\omega_{i}^{510}=\omega_{i}^{38}=\omega_{i}^{47}=0
$$

and finally the equality of:

$$
\begin{aligned}
& \left(\omega_{i}\right)^{122 \dot{2}}=\left(\omega_{i}^{36}-\omega_{i}^{310}\right)-i\left(\omega_{i}^{46}-\omega_{i}^{4}{ }^{10}\right), \\
& \left(\omega_{i}\right)^{212 \dot{2}}=\left(\omega_{i}^{36}-\omega_{i}^{310}\right)+i\left(\omega_{i}^{46}-\omega_{i}^{410}\right), \\
& \left(\omega_{i}\right)^{221 \dot{2}}=-\omega_{i}^{57}+i \omega_{i}^{58}
\end{aligned}
$$

implies:

$$
\omega_{i}^{46}=\omega_{i}^{410}=\omega_{i}^{58}=\omega_{i}^{36}=0, \omega_{i}^{57}=\omega_{i}^{310}-\omega_{i}^{36}
$$

One thus gets:

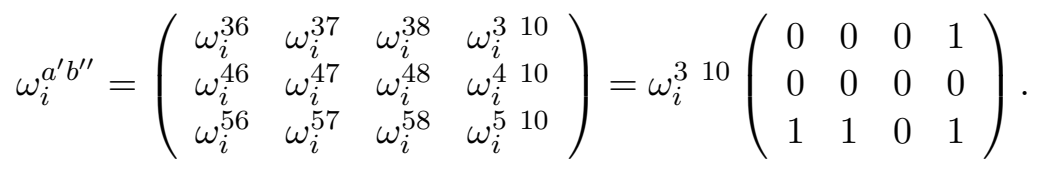

Hence, for associative three-folds, the number of independent components in the off-diagonal spin-connection is one: $\omega_{i}^{3}{ }^{10}$. We will set it to zero. The off-diagonal spin-connection component being set to zero towards the end of section 3 , is a consequence of the fact that for associative three-folds embedded in a $G_{2}$-manifold (See [14]), as considered in our work, (i) the self-dual piece of the connection on the normal bundle, " $\omega_{\perp}^{+}$", is unconstrained, and (ii) the connection on the tangent bundle, " $\omega_{\|}$" gets identified with the anti-self dual connection on normal bundle " $\omega_{\perp}^{-}$" - this is what was used in section 3 . These follow from the covariant constancy of a $G_{2}$ spinor and describe the decomposition of the adjoint representation of $G_{2} \subset \operatorname{Spin}(7)$ under $S O(3) \oplus S O(4)$. The aforementioned identification of spin connections is standard to "topological twisting" (See [14] and references therein) - one must keep in mind that one of the results of [14] is that the low energy fluctuations of an M2-brane wrapped around a supersymmetric three-fold is described by a three dimensional topological field thory. The results of this section will be utilized when evaluating the Seeley de-Witt coefficients for the Dirac-type operator $\mathcal{O}_{3}$ in the next section.

\section{Heat kernel Asymptotics of MQCD-like Supersymmetric Three-Fold embedded in $G_{2}$ Seven-Fold}

This section forms the core of the new results in this paper. We begin with a discussion of supersymmetric three-folds relevant to domain walls in MQCD and show that a certain infinite series, in the limit of a small complex constant $\zeta$, can in fact be summed to give a closed expression. We then proceed with the heat kernel asymptotics analysis by discussing the evaluation of the generalized zeta and eta function Seeley de-Witt coefficients relevant to the Laplace-type operator $\mathcal{O}_{1}$ and the Dirac-type operator $\mathcal{O}_{3}$. The main 
result that we get is (a) vanishing of the generalized eta function coefficients, and (b) a perfect match between the generalized zeta function coefficients for $\mathcal{O}_{1}$ and $\mathcal{O}_{3}^{2}$ - all calculations are done up to $\mathcal{O}\left(\zeta^{2}\right)$. The importance of these results was commented upon in the introduction and we make more comments in the section on conclusion. As an aside, we discuss a possible connection between the antiholomorphic involution relevant to Joyce's construction of "barely $G_{2}$ manifolds" from Calabi-Yau three-folds, and a similar involution symmetry of the supersymmetric three-fold - this three-fold turns out not to be a threecyle, but a three-fold with boundary (this necessitates the discussion of, both, bulk and boundary Seeley de-Witt coefficients).

In MQCD [2], discrete chiral symmetry breaking results in the formation of domain wall separating different vacua, whose world-volume is topologically given by $R^{3}\left(x^{0,1,2}\right) \times S\left(x^{3,4,5}\right)$, where $S$ is a supersymmetric three-fold embedded in a $G_{2}$-manifold that is topologically $\mathbf{R}\left(x^{3}\right) \times \mathbf{R}^{5}\left(x^{4,5,6,7,8}\right) \times S^{1}\left(x^{10}\right)$ Complexifying the coordinates, $v=x^{4}+i x^{5}, w=x^{7}+i x^{8}, s=x^{6}+i x^{10}, t=e^{-s}$, the boundary condition on $S$ is that as $x^{3} \rightarrow-\infty, S \rightarrow \mathbf{R} \times \Sigma$ and as $x^{3} \rightarrow \infty, S \rightarrow \mathbf{R} \times \Sigma^{\prime}$, where $\Sigma: w=$ $\zeta v^{-1}, t=v^{n}$ and $\Sigma^{\prime}: w=e^{\frac{2 \pi i}{n}} \zeta v^{-1}, t=v^{n}$. The calibration for $G_{2}$ manifolds can be written as: $\Phi=e^{123}+e^{136}+e^{145}+e^{235}-e^{246}+e^{347}+e^{567}\left(e^{i j k} \equiv e^{i} \wedge e^{j} \wedge e^{k}\right)$, and then the supersymmetric three-fold embedded in the $G_{2}$-manifold will be given as: $w=w\left(x^{3}, v, \bar{v}\right), s=s\left(x^{3}, v, \bar{v}\right)$. Then, defining the embedding as $x^{6}=A(x, y, z), x^{7}=C(x, y, z), x^{8}=D(x, y, z), x^{10}=B(x, y, z), x, y, z$ being the $M 2$-brane world-volume coordinates, the condition for supersymmetric cycle: $\left.\Phi\right|_{S}=\sqrt{g} d x^{3} \wedge d x^{4} \wedge d x^{5}$, after further relabeling $x^{3,4,5}$ as $z, x, y$ and after assuming: $\partial_{x} A=\partial_{y} B, \partial_{y} A=-\partial_{x} B$ (三 Cauchy-Riemann condition), translates to give:

$$
\begin{aligned}
& {\left[\partial_{x} A \partial_{z} A-\partial_{y} \partial_{z} B+\partial_{x} C \partial_{z} C-\partial_{y} C \partial_{z} D\right]^{2}+\left[\partial_{y} A \partial_{z} A+\partial_{x} A \partial_{z} B+\partial_{y} C \partial_{z} C+\partial_{x} C \partial_{z} D\right]^{2}} \\
& =\left[1+\left(\partial_{x} A\right)^{2}+\left(\partial_{y} A\right)^{2}+\left(\partial_{x} C\right)^{2}+\left(\partial_{y} C\right)^{2}\right]\left[\left(\partial_{z} A\right)^{2}+\left(\partial_{z} B\right)^{2}+\left(\partial_{z} C\right)^{2}+\left(\partial_{z} D\right)^{2}\right] .
\end{aligned}
$$

The ansatz to solve (25) for the embedding of the supersymmetric 3 -cycle in the $G_{2}$-manifold, taken in [1] was :

$$
\begin{aligned}
& v=\left[e^{\frac{y_{1}}{2}}+\sum_{m=1}^{\infty}\left(\frac{1}{2 \cosh y_{3}}\right)^{2 m} f_{2 m}\left(y_{1}\right)\right] e^{i y_{2}}, \\
& w=-\zeta \tanh y_{3}\left[e^{-\frac{y_{1}}{2}}+\sum_{m=1}^{\infty}\left(\frac{1}{2 \cosh y_{3}}\right)^{2 m} g_{2 m}\left(y_{1}\right)\right] e^{-i y_{1}}, \\
& s=-y_{1}-\sum_{m=1}^{\infty}\left(\frac{1}{2 \cosh y_{3}}\right)^{2 m} h_{2 m}\left(y_{1}\right)-2 i y_{2},
\end{aligned}
$$

where for the $S U(2)$ group, $f_{2 m}, g_{2 m}, h_{2 m}$ can be complex, but were taken to be real in [1]. The condition for getting a supersymmetric 3-cycle implemented by ensuring that the pull-back of the calibration $\Phi$ to the world volume of the 3-cycle is identical to the volume form on the 3-cycle, gives recursion relations between the coefficients $f_{2 m}$ and $g_{2 m}$, by setting $h_{2 m}=0$, e.g. for $m=1$, as shown in [1],

$$
\begin{aligned}
& -\zeta e^{-\frac{y_{1}}{2}} \partial_{1} f_{2}+\left(2 e^{\frac{y_{1}}{2}}+\frac{\zeta}{2} e^{-\frac{y_{1}}{2}}\right) f_{2}-\zeta e^{\frac{y_{1}}{2}} \partial_{1} g_{2}-\left(2 \zeta^{2} e^{\frac{-y_{1}}{2}}+\frac{\zeta}{2} e^{\frac{y_{1}}{2}}\right) g_{2}=-4 \zeta^{2} e^{\frac{-y_{1}}{2}}, \\
& -\left(\zeta^{2} e^{-y_{1}}+4\right) f_{2}+2 \zeta \partial_{1} g_{2}-\left(\zeta^{2}-\zeta\right) g_{2}=-2 \zeta^{2} e^{\frac{-y_{1}}{2}} .
\end{aligned}
$$

One can substitute for $f_{2}$ from the second equation and get a second order differential equation for $g_{2}$. However, it is shown that in the limit $\zeta \rightarrow 0$, one can consistently set $f_{2 m}=h_{2 m}=0, m \geq 1$. Further, 
surprisingly, as perhaps missed to be noticed in [1], one also gets the following differential equation for all $g_{2 m}$ 's, $m \geq 1$ :

$$
2 \partial_{1} g_{2 m}+g_{2 m}=\mathcal{O}(\zeta) \rightarrow 0
$$

implying

$$
g_{2 m}=e^{\frac{-x}{2}}, m \geq 1
$$

Hence, (26) becomes:

$$
\begin{aligned}
& v(x, z)=e^{\frac{x}{2}+i y}, \\
& w(x, z)=-\zeta \frac{\tanh (z) e^{\frac{-x}{2}}}{1-\left(\frac{\operatorname{sech}(z)}{2}\right)^{2}} e^{-i y}, \\
& s(x, y)=-x-2 i y .
\end{aligned}
$$

One thus gets a convergent solution, unlike the case for finite $\zeta$ as pointed out in [21].

We now consider an M-theory instanton obtained by wrapping a Euclidean $M 2$-brane around the supersymmetric noncompact three-fold embedded in a $G_{2}$-manifold relevant to MQCD, and perform a heat kernel asymptotics analysis for the membrane instanton superpotential as obtained in (9) and explore the possibility of cancelations between the bosonic and fermionic determinants. For bosonic determinants $\operatorname{det} A_{b}$, the function that is relevant is the generalized zeta function, $\zeta\left(s \mid A_{b}\right)$, and that for fermionic determinants $\operatorname{det} A_{f}$, the function that is additionally relevant is the generalized eta function, $\eta\left(s \mid A_{f}\right)$. The integral representation of the former involves $\operatorname{Tr}\left(e^{-t A_{b}}\right)$, while that for the latter involves $\operatorname{Tr}\left(A e^{-t A^{2}}\right)$ (See [19]):

$$
\zeta\left(s \mid A_{b}\right)=\frac{1}{\Gamma(2 s)} \int_{0}^{\infty} d t t^{s-1} \operatorname{Tr}\left(e^{-t A_{b}}\right) ; \eta\left(s \mid A_{f}\right)=\frac{1}{\Gamma\left(\frac{s+1}{2}\right)} \int_{0}^{\infty} d t t^{\frac{s+1}{2}} \operatorname{Tr}\left(A_{f} e^{-t A_{F}^{2}}\right),
$$

where to get the UV-divergent contributions, one looks at the $t \rightarrow 0$ limit of the two terms. To be more precise (See [20])

$$
\begin{aligned}
& \text { lndet } A_{b}=-\left.\frac{d}{d s} \zeta\left(s \mid A_{b}\right)\right|_{s=0} \\
& =-\left.\frac{d}{d s}\left(\frac{1}{\Gamma(s)} \int_{0}^{\infty} d t t^{s-1} \operatorname{Tr}\left(e^{-t A_{b}}\right)\right)\right|_{s=0} \\
& \operatorname{lndet} A_{f}=-\left.\left.\frac{1}{2} \frac{d}{d s} \zeta\left(s \mid A_{f}^{2}\right)\right|_{s=0} \mp \frac{i \pi}{2} \eta\left(s \mid A_{f}\right)\right|_{s=0} \pm\left.\frac{i \pi}{2} \zeta\left(s \mid A_{f}^{2}\right)\right|_{s=0} \\
& =\left.\left.\left[-\frac{1}{2} \frac{d}{d s} \pm \frac{i \pi}{2}\right]\left(\frac{1}{\Gamma(s)} \int_{0}^{\infty} d t t^{s-1} \operatorname{Tr}\left(e^{-t A_{f}^{2}}\right)\right)\right|_{s=0} \mp \frac{i \pi}{2} \frac{1}{\Gamma\left(\frac{s+1}{2}\right)} \int_{0}^{\infty} d t t^{\frac{s+1}{2}-1} \operatorname{Tr}\left(A_{f} e^{-t A_{f}^{2}}\right)\right|_{s=0},
\end{aligned}
$$

where the $\mp$ sign in front of $\eta(0)$, a non-local object, represents an ambiguity in the definition of the determinant. The $\zeta\left(0 \mid A_{f}^{2}\right)$ term can be reabsorbed into the contribution of $\zeta^{\prime}\left(0 \mid A_{f}^{2}\right)$, and hence will be dropped below. Here $\operatorname{Tr} \equiv \int d x\langle x|\ldots| x\rangle \equiv \int d x \operatorname{tr}(\ldots)$. The idea is that if one gets a match in the Seeley de Witt coefficients for the bosonic and fermionic determinants, implying equality of UV-divergence, this is indicative of a possible complete cancelation. 
The heat kernel expansions for the bosonic and fermionic determinants[15], in three dimensions, are given by:

$$
\operatorname{tr}\left(e^{-t A_{b}}\right)=\sum_{n=0}^{\infty} e_{n}\left(x, A_{b}\right) t^{\frac{(n-3)}{2}}, \operatorname{tr}\left(A_{f} e^{-t A_{f}^{2}}\right)=\sum_{n=0}^{\infty} a_{n}\left(x, A_{f}\right) t^{\frac{(n-4)}{2}}
$$

For Laplace-type operators $A_{b}$ and $A_{f}^{2}$ (the $b$ implies bosonic and $f$ implies fermionic), the non-zero coefficients in the bulk, are determined to be the following:

$$
e_{0}\left(x, A_{b}\right)=(4 \pi)^{-\frac{3}{2}} I d, e_{2}\left(x, A_{b}\right)=(4 \pi)^{-\frac{3}{2}}\left[\alpha_{1} E+\alpha_{2} R I d\right],
$$

where $R$ is the Ricci scalar constructed from suitable pull-backs of the metric and affine connection, $\alpha_{i}$ 's are constants, $I d$ is the identity that figures with the scalar leading symbol in the Laplace-type operator $A_{b}$ (See $[15])$, and

$$
\begin{aligned}
& E \equiv B-G^{i j}\left(\partial_{i} \omega_{j}+\omega_{i} \omega_{j}-\omega_{k} \Gamma_{i j}^{k}\right), \\
& A_{b} \equiv-\left(G^{i j} I d \partial_{i} \partial_{j}+A^{i} \partial_{i}+B\right), \\
& \omega_{i}=\frac{G_{i j}\left(A^{j}+G^{k l} \Gamma_{k l}^{j} I d\right)}{2} .
\end{aligned}
$$

For matrix-valued $E$, as will be the case for the Laplace-type $\mathcal{O}_{1}$ and the Dirac-type $\mathcal{O}_{3}$ in this paper, it is understood that one has to take a trace.

From the expressions of $\mathcal{O}_{1,2,3}$, one sees that the effective pullback of the metric (which gets used in, e.g., (34) and (35)) on to the world volume of the supersymmetric 3-cycle is given by:

$$
G_{i j}=\frac{g_{i j}}{\sqrt{g}}=\left(\begin{array}{ccc}
G_{11} & 0 & G_{13} \\
0 & G_{22} & 0 \\
G_{13} & 0 & G_{23}
\end{array}\right)
$$

where the components are either $\mathcal{O}(1)+\mathcal{O}\left(\zeta^{2}\right)$, e.g.,

$G_{11}=\frac{\sqrt{\left(4+e^{\frac{x}{2}}\right)\left(4+e^{x}\right)}}{2\left(4+e^{x}\right)}+\frac{4\left(\frac{2 \tanh (z)^{2}}{\left(-4+\operatorname{sech}(z)^{2}\right)^{2}}-\frac{\frac{\left(4+e^{\frac{x}{2}}\right) \tanh (z)^{2}}{\left(-4+\operatorname{sech}(z)^{2}\right)^{2}}+\left(4+e^{x}\right)\left(\frac{\left(4+e^{\frac{x}{2}}\right)(2+\cosh (2 z))^{2}}{(1+2 \cosh (2 z))^{4}}+\frac{\tanh (z)^{2}}{\left(-4+\operatorname{sech}(z)^{2}\right)^{2}}\right)}{4+e^{x}}\right) \zeta^{2}}{e^{x} \sqrt{\left(4+e^{\frac{x}{2}}\right)\left(4+e^{x}\right)}}+O(\zeta)^{3}$,

or $\mathcal{O}\left(\zeta^{2}\right)$, e.g.

$$
G_{13}=\frac{-8(2+\cosh (2 z)) \sinh (2 z) \zeta^{2}}{e^{x} \sqrt{\left(4+e^{\frac{x}{2}}\right)\left(4+e^{x}\right)}(1+2 \cosh (2 z))^{3}}+O(\zeta)^{3}
$$


The corresponding vielbeins (which get used in the evaluation of spin connections relevant to (39)-(41)) are therefore given by:

$$
e_{i}^{a^{\prime}}=\left(\begin{array}{ccc}
0 & e_{1}^{2} & e_{1}^{3} \\
0 & e_{2}^{1} & e_{2}^{3} \\
e_{3}^{1} & 0 & 0
\end{array}\right)
$$

where all components are of $\mathcal{O}(1)+\mathcal{O}\left(\zeta^{2}\right)$, e.g.,

$$
e_{1}^{2}=\frac{e^{\frac{x}{2}} \cos (y)}{\sqrt{2}\left(\left(4+e^{\frac{x}{2}}\right)\left(4+e^{x}\right)\right)^{\frac{1}{4}}}-\frac{2 \sqrt{2} \cos (y)\left(\frac{\left(4+e^{\frac{x}{2}}\right) \tanh (z)^{2}}{\left(-4+\operatorname{sech}(z)^{2}\right)^{2}}+\left(4+e^{x}\right)\left(\frac{\left(4+e^{\frac{x}{2}}\right)(2+\cosh (2 z))^{2}}{(1+2 \cosh (2 z))^{4}}+\frac{\tanh (z)^{2}}{\left(-4+\operatorname{sech}(z)^{2}\right)^{2}}\right)\right) \zeta^{2}}{e^{\frac{x}{2}}\left(\left(4+e^{\frac{x}{2}}\right)\left(4+e^{x}\right)\right)^{\frac{5}{4}}}
$$

and

$$
\mathcal{E}_{i}{ }^{a^{\prime \prime}}=\left(\begin{array}{cccc}
\mathcal{E}_{1}{ }^{1} & \mathcal{E}_{1}{ }^{2} & \mathcal{E}_{1}{ }^{3} & 0 \\
0 & \mathcal{E}_{2}{ }^{2} & \mathcal{E}_{2}{ }^{3} & \mathcal{E}_{2}{ }^{4} \\
0 & \mathcal{E}_{3}{ }^{2} & \mathcal{E}_{3}{ }^{3} & 0
\end{array}\right)
$$

where the components are either of $\mathcal{O}(1)+\mathcal{O}\left(\zeta^{2}\right)$, e.g.,

$\mathcal{E}_{1}{ }^{1}=-\left(\left(1+\frac{e^{\frac{x}{2}}}{4}\right)\left(4+e^{x}\right)\right)^{-\left(\frac{1}{4}\right)}+\frac{4 \sqrt{2}\left(\frac{\left(4+e^{\frac{x}{2}}\right) \tanh (z)^{2}}{\left(-4+\operatorname{sech}(z)^{2}\right)^{2}}+\left(4+e^{x}\right)\left(\frac{\left(4+e^{\frac{x}{2}}\right)(2+\cosh (2 z))^{2}}{(1+2 \cosh (2 z))^{4}}+\frac{\tanh (z)^{2}}{\left(-4+\operatorname{sech}(z)^{2}\right)^{2}}\right)\right) \zeta^{2}}{e^{x}\left(\left(4+e^{\frac{x}{2}}\right)\left(4+e^{x}\right)\right)^{\frac{5}{4}}}+O(\zeta)^{3}$ or of $\mathcal{O}(\zeta)$, e.g.,

$$
\mathcal{E}_{1}^{2}=\frac{-2 \cos (y) \tanh (z) \zeta}{e^{\frac{x}{2}}\left(\left(1+\frac{e^{\frac{x}{2}}}{4}\right)\left(4+e^{x}\right)\right)^{\frac{1}{4}}\left(-4+\operatorname{sech}(z)^{2}\right)}+O(\zeta)^{3} .
$$

The affine connection (relevant for evaluation of $\omega_{i}^{b}$ - see (42) - which gets used in the evaluation of $e_{n} \mathrm{~s}$ via (35) and (34)) for $G_{i j}$ are given as under:

$$
\Gamma_{j k}^{i}=\left(\begin{array}{ccc}
\Gamma_{11}^{1} & O(\zeta)^{4} & \Gamma_{13}^{1} \\
O(\zeta)^{4} & \Gamma_{22}^{1} & O(\zeta)^{4} \\
O(\zeta)^{4} & \Gamma_{32}^{1} & \Gamma_{33}^{1} \\
& & \\
O(\zeta)^{4} & \Gamma_{12}^{2} & O(\zeta)^{4} \\
\Gamma_{21}^{2} & O(\zeta)^{4} & \Gamma_{23}^{2} \\
O(\zeta)^{4} & \Gamma_{32}^{2} & O(\zeta)^{4} \\
& & \\
\Gamma_{11}^{3} & O(\zeta)^{4} & \Gamma_{13}^{3} \\
O(\zeta)^{4} & \Gamma_{22}^{3} & O(\zeta)^{4} \\
\Gamma_{31}^{3} & O(\zeta)^{4} & \Gamma_{33}^{3}
\end{array}\right)
$$


Using $\Gamma_{j k}^{i}$, one can then evaluate the various components of the curvature tensor, the non-zero being: $R_{121}^{2}, R_{131}^{3}, R_{123}^{2}, R_{232}^{3}, R_{323}^{2}, R_{343}^{4}$. Using these, one evaluates the non-zero components of the Ricci tensor: $R_{11}, R_{13}, R_{22}$ and $R_{33}$ which gives the Ricci scalar (relevant for evaluation of the Seeley de-Witt coefficient $e_{2}\left(x, \mathcal{O}_{1}\right.$ or 2 or $\left.\mathcal{O}_{3}^{2}\right)$ - see $\left.(34)\right)$ :

$$
R=\mathcal{O}(1)+\mathcal{O}\left(\zeta^{2}\right)+\mathcal{O}\left(\zeta^{4}\right),
$$

where the first two terms, which can be easily calculated, are relevant to the order we are working. As the actual expression for $R$ does not explicitly get used in this paper and is also very long, we skip giving the same.

The three-fold is topologically $M_{3}=\mathbf{R} \times[0,1] \times S^{1}$, implying that it has a boundary which is given by $\partial M_{3}=\left(R \times S^{1}, 0\right) \cup\left(R \times S^{1}, 1\right)$. Note that configurations involving branes wrapping noncompact cycles have been studied earlier - see [10]. The three-fold is given by the set of following equations:

$$
\begin{aligned}
& \left(x^{4}\right)^{2}+\left(x^{5}\right)^{2}=e^{-x^{6}}, \\
& \frac{x^{5}}{x^{4}}=-\tan \left(\frac{x^{10}}{2}\right), \\
& \frac{x^{8}}{x^{7}}=\tan \left(\frac{x^{10}}{2}\right), \\
& \left(x^{7}\right)^{2}+\left(x^{8}\right)^{2}=\frac{\zeta^{2} e^{x^{6}}\left(2-\frac{3 x^{8} e^{-\frac{x^{6}}{2}} \sec \left(\frac{x^{10}}{2}\right)}{4 \zeta}-2 \sqrt{\left.1-\frac{3 x^{8} e^{-\frac{x^{6}}{2}} \sec \left(\frac{x^{10}}{2}\right)}{4 \zeta}\right)}\right.}{4\left(3-\frac{\left(2-\frac{3 x^{8} e^{-\frac{x^{6}}{2}} \sec \left(\frac{x^{10}}{2}\right)}{4 \zeta}-2 \sqrt{\left.1-\frac{3 x^{8} e^{-\frac{x^{6}}{2}} \sec \left(\frac{x^{10}}{2}\right)}{4 \zeta}\right)}\right.}{4}\right)^{2}} .
\end{aligned}
$$

One notices the following $\mathbf{Z}_{2}$ symmetry of (36):

$$
x_{4,7,6} \rightarrow x_{4,7,6} ; x_{5,8,10} \rightarrow-x_{5,8,10} ; \zeta \rightarrow-\zeta .
$$

Notice that under the above antiholomorphic involution: $J=d u \wedge d \bar{u}+d v \wedge d \bar{v}+d s \wedge d \bar{s}$ is reflected, and $\Omega=d u \wedge d v \wedge d s$ is complex conjugated. This is related to the involution used in the construction of a $G_{2}$ manifold from a Calabi-Yau three-fold using the Joyce's prescription: $\frac{C Y_{3} \times S^{1}}{\mathbf{Z}_{2}}$.

Given that the supersymmetric three-fold $M_{3}$ has a nontrivial boundary, in addition to the bulk Seeley de-Witt coefficients given by (34), one also needs to evaluate boundary Seeley-de Witt coefficients. The latter for $M_{3}$ of (36) are given by (See [15]):

$$
\begin{aligned}
& a_{1}^{\partial M_{3}}=\frac{1}{4 \pi}\left(\frac{\Gamma\left(\frac{3}{2}\right)}{\Gamma\left(\frac{1}{2}\right) \Gamma(2)}-1\right) \operatorname{tr} \mathbf{1}, \\
& a_{2}^{\partial M_{3}}=\left.\frac{1}{(4 \pi)^{\frac{3}{2}}} \frac{1}{3}\left(1-\frac{3}{4} \pi \frac{\Gamma\left(\frac{3}{2}\right)}{\Gamma\left(\frac{1}{2}\right) \Gamma(2)}\right) \Pi_{i i}\right|_{\partial M_{3}},
\end{aligned}
$$

$\Pi_{i i}$ being the trace of the second fundamental form. The second fundamental form is given by: $\Pi_{i j}=$ $\left\langle n, \nabla u_{i} v_{j}\right\rangle$, where the tangent vectors $u_{i}=\frac{\partial x^{m}}{\partial y^{i}} \partial_{m}$, and similarly for $v_{j}$, where $m$ takes values $3,6,10$. For $\partial M_{3}, M_{3}$ being given by (36), $\Pi_{i j}=n^{x^{6}} \frac{\partial^{2} x^{6}}{\partial y_{i} \partial y_{j}}$, which for the embedding $x^{6}=-y^{1}$, vanishes.

We now proceed with the evaluation of the bulk Seeley de-Witt coefficients as given in (34). For this we would first evaluate $A_{b}^{i} \mathrm{~s}$ (the " $b$ " implies relevant to bosonic operators) utilizing the results for the vielbeins 
obtained earlier in this section. This is done in equations (39)-(41). Using the results for $A_{b}^{i} \mathrm{~s}$, we would then calculate $\omega_{i}^{b}$ s. The same is done in equation (42) for $\omega_{1}^{b}$ - one can similarly evaluate $\omega_{2}^{b}$ and $\omega_{3}^{b}$. We would then be able to calculate $E$, which would enable us to evaluate $e_{n}$ s. This is done in equations (43)-(46).

For the operator $\mathcal{O}_{1}$, the expressions for $A_{b}^{i}$ (see (8) and (35) ) are given as:

$$
\begin{aligned}
& A_{b}^{1}=\delta_{u v} G^{1 j}\left(\left.\omega_{j}\right|_{M_{3}}+\left.\omega_{j}\right|_{N\left(M_{3}\right) \hookrightarrow X_{G_{2}}}\right) \\
& =\left(\begin{array}{ccccccc}
0 & a_{12} & a_{13} & 0 & 0 & 0 & 0 \\
-a_{12} & 0 & a_{23} & 0 & 0 & 0 & 0 \\
-a_{13} & -a_{23} & 0 & 0 & 0 & 0 & 0 \\
0 & 0 & 0 & 0 & a_{45} & a_{46} & O(\zeta)^{2} \\
0 & 0 & 0 & -a_{45} & 0 & a_{56} & a_{57} \\
0 & 0 & 0 & -a_{46} & -a_{56} & 0 & a_{67} \\
0 & 0 & 0 & O(\zeta)^{2} & -a_{57} & -a_{67} & 0
\end{array}\right)
\end{aligned}
$$

( $u, v$ index $\mathbf{R}^{4}$-valued coordinates and $N\left(M_{3}\right)$ is the normal bundle to $M_{3}$ ). Similarly,

$$
\begin{aligned}
& A_{b}^{2}=\delta_{u v} G^{2 j}\left(\left.\omega_{j}\right|_{M_{3}}+\left.\omega_{j}\right|_{N\left(M_{3}\right) \hookrightarrow X_{G_{2}}}\right) \\
& =\left(\begin{array}{ccccccc}
0 & b_{12} & b_{13} & 0 & 0 & 0 & 0 \\
-b_{12} & 0 & b_{23} & 0 & 0 & 0 & 0 \\
-b_{13} & -b_{23} & 0 & 0 & 0 & 0 & 0 \\
0 & 0 & 0 & 0 & b_{45} & b_{46} & b_{47} \\
0 & 0 & 0 & -b_{45} & 0 & b_{56} & b_{57} \\
0 & 0 & 0 & -b_{46} & -b_{56} & 0 & b_{67} \\
0 & 0 & 0 & -b_{47} & -b_{57} & -b_{67} & 0
\end{array}\right),
\end{aligned}
$$

and

$$
\begin{gathered}
A_{b}^{3}=\delta_{u v} G^{3 j}\left(\left.\omega_{j}\right|_{M 3}+\left.\omega_{j}\right|_{\left.N\left(M_{3}\right) \hookrightarrow X_{G_{2}}\right)}\right) \\
=\left(\begin{array}{ccccccc}
0 & c_{12} & c_{13} & 0 & 0 & 0 & 0 \\
-c_{12} & 0 & c_{23} & 0 & 0 & 0 & 0 \\
-c_{13} & -c_{23} & 0 & 0 & 0 & 0 & 0 \\
0 & 0 & 0 & 0 & c_{45} & c_{46} & O(\zeta)^{2} \\
0 & 0 & 0 & -c_{45} & 0 & c_{56} & O(\zeta)^{2} \\
0 & 0 & 0 & -c_{46} & -c_{56} & 0 & O(\zeta)^{2} \\
0 & 0 & 0 & O(\zeta)^{2} & O(\zeta)^{2} & O(\zeta)^{2} & 0
\end{array}\right),
\end{gathered}
$$

where the various non-zero elements can easily be worked out.

To evaluate the $\zeta$ Seeley de-Witt coefficients, one needs to evaluate $\omega_{i}^{b}$. The expressions for $\omega_{i}^{b}$ are given as:

$$
\omega_{1}^{b}=\frac{G_{1 j}}{2}\left(A_{b}^{j}+G^{k l} \Gamma_{k l}^{j} \mathbf{1}_{7}\right)
$$




$$
=\left(\begin{array}{ccccccc}
w_{111} & w_{112} & w_{113} & O(\zeta)^{4} & O(\zeta)^{4} & O(\zeta)^{4} & O(\zeta)^{4} \\
w_{121} & w_{122} & w_{123} & O(\zeta)^{4} & O(\zeta)^{4} & O(\zeta)^{4} & O(\zeta)^{4} \\
w_{131} & w_{132} & w_{133} & O(\zeta)^{4} & O(\zeta)^{4} & O(\zeta)^{4} & O(\zeta)^{4} \\
O(\zeta)^{4} & O(\zeta)^{4} & O(\zeta)^{4} & w_{144} & w_{145} & w_{146} & \left.O(\zeta)^{2}\right) \\
O(\zeta)^{4} & O(\zeta)^{4} & O(\zeta)^{4} & w_{154} & w_{155} & w_{156} & w_{157} \\
O(\zeta)^{4} & O(\zeta)^{4} & O(\zeta)^{4} & w_{164} & w_{165} & w_{166} & w_{167} \\
O(\zeta)^{4} & O(\zeta)^{4} & O(\zeta)^{4} & O(\zeta)^{2} & w_{175} & w_{176} & w_{177}
\end{array}\right)
$$

One can similarly evaluate expressions for $\omega_{2,3}^{b}$, using which one can evaluate $E\left(\mathcal{O}_{1}\right)=E_{1}^{b}+E_{2}^{b}+E_{3}^{b}$, where

$$
\begin{aligned}
& E_{1}^{b}=-G^{i j} \partial_{i} \omega_{j}^{b} \\
& =\left(\begin{array}{ccccccc}
E_{111} & E_{112} & E_{113} & O(\zeta)^{4} & O(\zeta)^{4} & O(\zeta)^{4} & O(\zeta)^{4} \\
E_{121} & E_{122} & E_{123} & O(\zeta)^{4} & O(\zeta)^{4} & O(\zeta)^{4} & O(\zeta)^{4} \\
E_{131} & E_{132} & E_{133} & O(\zeta)^{4} & O(\zeta)^{4} & O(\zeta)^{4} & O(\zeta)^{4} \\
O(\zeta)^{4} & O(\zeta)^{4} & O(\zeta)^{4} & E_{144} & E_{145} & E_{146} & O(\zeta)^{2} \\
O(\zeta)^{4} & O(\zeta)^{4} & O(\zeta)^{4} & E_{154} & E_{155} & E_{156} & E_{157} \\
O(\zeta)^{4} & O(\zeta)^{4} & O(\zeta)^{4} & E_{164} & E_{165} & E_{166} & E_{167} \\
O(\zeta)^{4} & O(\zeta)^{4} & O(\zeta)^{4} & O(\zeta)^{2} & E_{175} & E_{176} & E_{177}
\end{array}\right),
\end{aligned}
$$

where the non-zero elements can be easily worked out. Similarly,

$$
\begin{aligned}
& E_{2}^{b}=-G^{i j} \omega_{i}^{b} \omega_{j}^{b} \\
& =\left(\begin{array}{ccccccc}
E_{211} & E_{212} & E_{213} & O(\zeta)^{8} & O(\zeta)^{8} & O(\zeta)^{8} & O(\zeta)^{8} \\
E_{221} & E_{222} & E_{223} & O(\zeta)^{8} & O(\zeta)^{8} & O(\zeta)^{8} & O(\zeta)^{8} \\
E_{231} & E_{232} & E_{233} & O(\zeta)^{8} & O(\zeta)^{8} & O(\zeta)^{8} & O(\zeta)^{8} \\
O(\zeta)^{8} & O(\zeta)^{8} & O(\zeta)^{8} & E_{244} & E_{245} & E_{246} & E_{247} \\
O(\zeta)^{8} & O(\zeta)^{8} & O(\zeta)^{8} & E_{254} & E_{255} & O(\zeta)^{4} & E_{257} \\
O(\zeta)^{8} & O(\zeta)^{8} & O(\zeta)^{8} & E_{264} & O(\zeta)^{4} & E_{266} & E_{267} \\
O(\zeta)^{8} & O(\zeta)^{8} & O(\zeta)^{8} & E_{274} & E_{275} & E_{276} & E_{277}
\end{array}\right)
\end{aligned}
$$

and

$$
\begin{aligned}
& E_{3}^{b}=G^{i j} \omega_{k}^{b} \Gamma_{i j}^{k} \\
& =\left(\begin{array}{ccccccc}
E_{311} & E_{312} & E_{313} & O(\zeta)^{4} & O(\zeta)^{4} & O(\zeta)^{4} & O(\zeta)^{4} \\
E_{321} & E_{322} & E_{323} & O(\zeta)^{4} & O(\zeta)^{4} & O(\zeta)^{4} & O(\zeta)^{4} \\
E_{331} & E_{332} & E_{333} & O(\zeta)^{4} & O(\zeta)^{4} & O(\zeta)^{4} & O(\zeta)^{4} \\
O(\zeta)^{4} & O(\zeta)^{4} & O(\zeta)^{4} & E_{344} & E_{345} & E_{346} & O(\zeta)^{2} \\
O(\zeta)^{4} & O(\zeta)^{4} & O(\zeta)^{4} & E_{354} & E_{355} & E_{356} & E_{357} \\
O(\zeta)^{4} & O(\zeta)^{4} & O(\zeta)^{4} & E_{364} & E_{365} & E_{366} & E_{367} \\
O(\zeta)^{4} & O(\zeta)^{4} & O(\zeta)^{4} & O(\zeta)^{2} & E_{375} & E_{376} & E_{377}
\end{array}\right) .
\end{aligned}
$$

One thus gets:

$$
\begin{aligned}
& \operatorname{tr}\left(E\left(\mathcal{O}_{1}\right)\right)=\operatorname{tr}\left(E_{1}^{b}+E_{2}^{b}+E_{3}^{b}\right)=\frac{\left(4+e^{x}\right)\left(-1536 e^{\frac{x}{2}}+2192 e^{x}+64 e^{\frac{3 x}{2}}+216 e^{2 x}-112 e^{\frac{5 x}{2}}+e^{3 x}\right)}{128\left(\left(4+e^{\frac{x}{2}}\right)\left(4+e^{x}\right)\right)^{\frac{5}{2}}}+ \\
& \frac{E \zeta^{2}}{128 e^{x}\left(\left(4+e^{\frac{x}{2}}\right)\left(4+e^{x}\right)\right)^{\frac{7}{2}}(1+2 \cosh (2 z))^{6}}+O\left(\zeta^{3}\right),
\end{aligned}
$$


where

$$
\begin{aligned}
& E \equiv\left(4+e^{x}\right)\left(4237426688+4268851200 e^{\frac{x}{2}}+4797884672 e^{x}+3473765744 e^{\frac{3 x}{2}}\right. \\
& +2023406480 e^{2 x}+1003298120 e^{\frac{5 x}{2}}+381310232 e^{3 x}+117349175 e^{\frac{7 x}{2}}+28347453 e^{4 x} \\
& +4231472 e^{\frac{9 x}{2}}+267264 e^{5 x}+4\left(617218048+628211712 e^{\frac{x}{2}}+705386752 e^{x}\right. \\
& +512375632 e^{\frac{3 x}{2}}+299013040 e^{2 x}+148217272 e^{\frac{5 x}{2}}+56510632 e^{3 x}+17391397 e^{\frac{7 x}{2}}+4212567 e^{4 x} \\
& \left.+635494 e^{\frac{9 x}{2}}+40704 e^{5 x}\right) \cosh (2 z)-4\left(554958848+551976960 e^{\frac{x}{2}}+620804864 e^{x}+447750848 e^{\frac{3 x}{2}}+260136512 e^{2 x}\right. \\
& \left.+129088208 e^{\frac{5 x}{2}}+48864080 e^{3 x}+15043796 e^{\frac{7 x}{2}}+3621852 e^{4 x}+532883 e^{\frac{9 x}{2}}+33024 e^{5 x}\right) \cosh (4 z) \\
& -726663168 \cosh (6 z)-733347840 e^{\frac{x}{2}} \cosh (6 z)-821714944 e^{x} \cosh (6 z)-596084096 e^{\frac{3 x}{2}} \cosh (6 z) \\
& -346978944 e^{2 x} \cosh (6 z)-172383936 e^{\frac{5 x}{2}} \cosh (6 z)-65568064 e^{3 x} \cosh (6 z) \\
& -20221080 e^{\frac{7 x}{2}} \cosh (6 z)-4890248 e^{4 x} \cosh (6 z)-728600 e^{\frac{9 x}{2}} \cosh (6 z) \\
& -46080 e^{5 x} \cosh (6 z)-25165824 \cosh (8 z)-30932992 e^{\frac{x}{2}} \cosh (8 z)-32834560 e^{x} \cosh (8 z)-25648768 e^{\frac{3 x}{2}} \cosh (8 z) \\
& -15232384 e^{2 x} \cosh (8 z)-7692928 e^{\frac{5 x}{2}} \cosh (8 z)-3094272 e^{3 x} \cosh (8 z)-975624 e^{\frac{7 x}{2}} \cosh (8 z) \\
& -247896 e^{4 x} \cosh (8 z)-41180 e^{\frac{9 x}{2}} \cosh (8 z)-3072 e^{5 x} \cosh (8 z)+2621440 \cosh (10 z)+2031616 e^{\frac{x}{2}} \cosh (10 z) \\
& +2505728 e^{x} \cosh (10 z)+1632320 e^{\frac{3 x}{2}} \cosh (10 z)+924608 e^{2 x} \cosh (10 z)+455648 e^{\frac{5 x}{2}} \cosh (10 z) \\
& +149152 e^{3 x} \cosh (10 z)+43780 e^{\frac{7 x}{2}} \cosh (10 z)+8748 e^{4 x} \cosh (10 z)+960 e^{\frac{9 x}{2}} \cosh (10 z) \\
& -131072 \cosh (12 z)-98304 e^{\frac{x}{2}} \cosh (12 z)-110848 e^{x} \cosh (12 z)-63984 e^{\frac{3 x}{2}} \cosh (12 z)-32528 e^{2 x} \cosh (12 z) \\
& \left.-12296 e^{\frac{5 x}{2}} \cosh (12 z)-5464 e^{3 x} \cosh (12 z)-1535 e^{\frac{7 x}{2}} \cosh (12 z)-469 e^{4 x} \cosh (12 z)\right)
\end{aligned}
$$

Despite the very complicated and long (46) and (47), we will see shortly that one gets a remarkable result, which is that the square of the relevant Dirac-type operator, " $A_{f}^{2}$ " ( " $f$ " denoting fermionic) - $\mathcal{O}_{3}^{2}$ - contributes precisely as " $A_{b}$ " $\left(\equiv \mathcal{O}_{1}\right)$ - see (57). This, in itself is a check of our lengthy spectral analysis, because (57) and the fact (which again we show momentarily) that the $\eta$-function contribution from $A_{f}$ vanishes, is something we had anticipated from supersymmetry arguments (given that one is dealing with a supersymmetric threefold for getting the membrane instanton), but the same was totally unobvious from a spectral analysis point of view.

We now do a heat-kernel asymptotics analysis of the fermionic determinant $\operatorname{det} \mathcal{O}_{3}$. The fermionic operator $\mathcal{O}_{3}$ can be expressed as:

$$
\mathcal{O}_{3} \equiv \sqrt{g} g^{i j} \Gamma_{j} D_{i}=\sqrt{g} g^{i j} \Gamma_{j}\left(\partial_{i}+\frac{1}{4} \omega_{i}^{a^{\prime} b^{\prime}} \Gamma_{a^{\prime} b^{\prime}}+\frac{1}{4} \omega_{i}^{a^{\prime} b^{\prime \prime}} \Gamma_{a^{\prime} b^{\prime \prime}}\right) \equiv G^{i j} \Gamma_{j} \partial_{i}-r,
$$

where

$$
G^{i j} \equiv \sqrt{g} g^{i j} ; r \equiv \frac{-1}{4} \sqrt{g} g^{i j} \Gamma_{j}\left(\omega_{i}^{a^{\prime} b^{\prime}} \Gamma_{a^{\prime} b^{\prime}}+\omega_{i}^{a^{\prime} b^{\prime \prime}} \Gamma_{a^{\prime} b^{\prime \prime}}\right)
$$

and using the results of section 3 , we set $\omega_{i}^{a^{\prime} b^{\prime \prime}}=0 . \mathcal{O}_{3}$ is of the Dirac-type as $\mathcal{O}_{3}^{2}$ is of the Laplace-type, as can be seen from the following:

$$
\mathcal{O}_{3}^{2} \equiv G^{i j} \partial_{i} \partial_{j}+A^{i} \partial_{i}+B, \text { where : }
$$




$$
\begin{aligned}
& G^{i j} \equiv \sqrt{g} g^{i j} \\
& A^{i} \equiv G^{l k} \Gamma_{l} \partial_{k}\left(G^{j i} \Gamma_{j}\right)+G^{j i} G^{l k} \Gamma_{j} \Gamma_{l} \omega_{k}+G^{k l} G^{j i} \Gamma_{l} \omega_{k} \Gamma_{j} \\
& B \equiv G^{i j} \Gamma_{i} \partial_{j}\left(G^{k l} \Gamma_{k} \omega_{l}\right)+G^{i j} G^{k l} \Gamma_{i} \omega_{j} \Gamma_{k} \omega_{l} .
\end{aligned}
$$

The remark regarding the dissimilar $\mathcal{O}_{1}$ and $\mathcal{O}_{3}^{2}$ in the introduction is justified by comparing (8) and (50). Now,

$$
\mathcal{O}_{3} \equiv G^{i j} \Gamma_{j} \nabla_{i}-\phi
$$

where $\phi \equiv r+\Gamma^{i} \omega_{i}$, and

$$
\omega_{l} \equiv \frac{G_{i l}}{2}\left(-\Gamma^{j} \partial_{j} \Gamma^{i}+\left\{r, \Gamma^{i}\right\}+G^{j k} \Gamma_{j k}^{i}\right) .
$$

The bulk Seeley-de Witt coefficients $a_{i}$ are given by (See [15]):

$$
\begin{aligned}
& a_{1}\left(x, G^{i j} \Gamma_{j} \nabla i-\phi\right)=-(4 \pi)^{-\frac{3}{2}} \operatorname{tr}(\phi) ; \\
& a_{3}\left(x, G^{i j} \Gamma_{j} \nabla_{i}-\phi\right)=-\frac{1}{6}(4 \pi)^{-\frac{3}{2}} \operatorname{tr}\left(\phi R+6 \phi \mathcal{E}-\Omega_{a^{\prime} b^{\prime} ; a^{\prime}} \Gamma_{b^{\prime}}\right),
\end{aligned}
$$

where

$$
\mathcal{E} \equiv-\frac{1}{2} \Gamma^{i} \Gamma^{j} \Omega_{i j}+\Gamma^{i} \phi_{; i}-\phi^{2}, \Omega_{i j} \equiv \partial_{i} \omega_{j}-\partial_{j} \omega_{i}+\left[\omega_{i}, \omega_{j}\right]
$$

and $\Omega_{a^{\prime} b^{\prime}}=e_{a^{\prime}}^{i} e_{b^{\prime}}^{j} \Omega_{i j}$. To ensure that $\mathcal{O}_{3}^{2}$ is a Laplace-type operator, $\Gamma_{i} \equiv \frac{1}{g^{\frac{1}{4}}} \partial_{i} X^{M} \Gamma_{M}, M$ indexing the eleven (Euclidean) dimensions and $\Gamma_{M}$ being the generators of $C l(0,11)$. The boundary $\eta$-function Seeley-de Witt coefficients (See [15]) are given as:

$$
\begin{aligned}
& a_{2}^{\partial M_{3}}=\left.\frac{1}{4 \pi}\left(-\frac{1}{4}\left(\frac{\Gamma\left(\frac{3}{2}\right)}{\Gamma\left(\frac{1}{2}\right) \Gamma(2)}-1\right) \phi-\frac{\frac{\Gamma\left(\frac{3}{2}\right)}{\Gamma\left(\frac{1}{2}\right) \Gamma(2)}}{4} \Gamma^{i} \omega_{i}\right)\right|_{\partial M_{3}}, \\
& a_{3}^{\partial M_{3}}=\left.\frac{-1}{3(4 \pi)^{\frac{3}{2}}} \phi_{; 3}\right|_{\partial M_{3} .}
\end{aligned}
$$

Using the generators of $C l(0,7)$ :

$$
\begin{aligned}
& \gamma_{1}=i \sigma^{2} \otimes \sigma^{1} \otimes \mathbf{1}_{2}, \\
& \gamma_{2}=i \sigma^{2} \otimes \sigma^{3} \otimes \mathbf{1}_{2}, \\
& \gamma_{3}=\mathbf{1}_{2} \otimes i \sigma^{2} \otimes \sigma^{1}, \\
& \gamma_{4}=\mathbf{1}_{2} \otimes i \sigma^{2} \otimes \sigma^{3}, \\
& \gamma_{5}=\sigma^{1} \otimes \mathbf{1}_{2} \otimes i \sigma^{2}, \\
& \gamma_{6}=\sigma^{3} \otimes \mathbf{1}_{2} \otimes i \sigma^{2}, \\
& \gamma_{7}=i \sigma^{2} \otimes i \sigma^{2} \otimes i \sigma^{2},
\end{aligned}
$$

one can construct generators of $C l(0,11)$ (See [14]) as:

$$
\begin{aligned}
& \Gamma_{a^{\prime}}=\sigma^{a^{\prime}} \otimes\left(-\sigma^{3}\right) \otimes \mathbf{1}_{8}, \\
& \Gamma_{a^{\prime \prime}}=\mathbf{1}_{2} \otimes i \sigma^{2} \otimes \gamma_{a^{\prime \prime}},
\end{aligned}
$$


One then sees that all the terms in the $\eta$ Seeley de-Witt coefficients for $\mathcal{O}_{3}$ are of the type $\operatorname{tr}$ (odd,even) $+\operatorname{tr}$ (even,odd) where one counts the (number of $\Gamma_{a^{\prime}}$ 's, number of $\Gamma_{a^{\prime \prime}}$ 's). Now, using (56), one can show that $\operatorname{tr}\left(\prod_{i=1}^{2 m+1} \Gamma_{a_{i}^{\prime}}\right)=\operatorname{tr}\left(\prod_{i=1}^{2 n+1} \prod_{j=1}^{2 m} \Gamma_{a^{\prime \prime}}\right)=0$. This implies that the bulk and boundary $\eta$ Seeley de-Witt coefficients for $\mathcal{O}_{3}$ vanish.

Further, one sees that $B$ is traceless. Analogous to the bosonic sector contribution, one can evaluate $A_{f}^{i}$ ( $f$ denoting fermionic contribution) and therefore calculate $\omega_{i}^{f}$ 's, and using the latter, one gets the incredible result

$$
\operatorname{tr}\left(E\left(\mathcal{O}_{3}\right)\right)=\operatorname{tr}\left(E\left(\mathcal{O}_{1}^{2}\right)\right)=(46) \&(47) ! ! !
$$

From equations (46) and (47), we see that we get a match for the Seeley de-Witt coefficients, for terms including $\mathcal{O}\left(\zeta^{2}\right)$ - in fact the non-triviality of the calculations seem to be the perfect match of $\mathcal{O}\left(\zeta^{2}\right)$ terms for the bosonic and fermionic fluctuations. From (30), one sees that the dependence of the embedding of the associative three-fold in the $G_{2}$-manifold is via the dependence of the same on $\zeta$ - setting $\zeta$ to zero is equivalent to the reduction of the world-volume integral $\int d^{3} z(\ldots)(s)=\int d x d y d z(\ldots)(x, y, z)$ to the world-line integral $\int d z(\ldots)$ corresponding to the $D$ 0-brane of type $I I A$ theory in the vanishing $M$-theory circle limit.

Further, using (32), one thus conjectures that:

$$
\frac{\ln \operatorname{det} \mathcal{O}_{3}}{\ln \operatorname{det} \mathcal{O}_{1}}=\frac{1}{2}
$$

implying that the noncompact instanton has a residual supersymmetry - arrived upon from a heat kernel asymptotics/spectral analysis point of view.

\section{Conclusion}

The Seeley de-Witt coefficients associated with the nonperturbative superpotential generated by an MQCDlike instanton configuration obtained by wrapping $M 2$-brane around a noncompact supersymmetric threefold embedded in a (noncompact) $G_{2}$-manifold relevant to MQCD, understood as the $M$ theory configuration dual to a type $I I B$ configuration compactified on a circle of vanishing radius, was considered in this paper ${ }^{5}$. The boundary $\eta$ Seeley de-Witt coefficients for the relevant fermionic operator vanish. Up to second order in a complex parameter that is part of the embedding of the aforementioned three-fold in the $G_{2}$ seven-fold, we get a perfect match between the Seeley de-Witt coefficients between the fermionic and one of the two bosonic determinants thereby strongly suggesting the presence of the expected surviving supersymmetry of the nonperturbative configurations in $M$-theory. From a spectral analysis point of view, the results themselves provide a remarkable check - in particular, if one looks at the extremely long and complicated expressions given in equations (46) and (47) for the Laplace-type operator $\mathcal{O}_{1}$, it is extremely nontrivial to see that one gets exactly the same expression for the Dirac-type operator $\mathcal{O}_{3}$ in equation (57). Further, this also shows that one might get quantum corrections from the uncancelled $\ln$ det $\mathcal{O}_{2}$ (at least in the static gauge used). One has also to appreciate that the quantities involved in the calculations, are not just pullback of the space-time metric and the Gamma matrices, but involve, e.g., pseudo-metrics (because of the extra square root of the pulled back metric).

\footnotetext{
${ }^{5}$ For a noncompact membrane instanton, what is more appropriate to be considered is $e^{\frac{1}{l_{11}^{3}} \operatorname{vol}(g)} \Delta W$ rather than $\Delta W-$ the former will be independent of the volume of the noncompact instanton.
} 
Given the direct-product topology $S \times[0,1]$ of the $M 2$-brane, one can ask the question what happens if the $M 2$-brane does in fact end on $M 5$-branes on the interval, or even $M 9$-branes. One would then have to deal with the contribution to the superpotential coming from the $M 5-M 5, M 5-M 9$ and $M 9-M 9$ open membrane instantons - the $M 9-M 9$ instantons, the $M$-theory analogues of world-sheet instantons, often sum up to zero (See [23] and references therein) however. A sketch of the relevant expressions in the context of heterotic $M$-theory is given in, e.g., [23]. In the context of plain $M$-theory on $G_{2}$-manifolds, the $M 5-M 5$ superpotential in the supegravity approximation, e.g., would be of the form: $e^{\left(X_{1}-X_{2}\right) \int_{S}\left(i \frac{\partial}{\partial X}{ }^{C+i J)}\right.}(\ldots)$, where $X_{i}$ is the complexified position of the $M 5$-branes obtained from the $M$-theory chiral two form (corresponding to a self-dual field strength on the $M 5$-brane world-volume) - See [14]. Based on arguments given in [23], one would guess (especially for "barely" $G_{2}$ manifolds) that supersymmetry requirements would be met if the $M$-theory circle direction is an appropriate function of the interval coordinate ( $x$ in our paper), and the other internal coordinates depend on $y$ and $z$ (of our paper).

The nonperturbative membrane instanton contribution to the superpotential can be compared with the complexified affine-Toda-like superpotential, generated by three-dimensional instantons (or four-dimensional monopoles) in the compactification of the $D=4, \mathcal{N}=1 \mathrm{SYM}$ on a circle to $D=3(\mathcal{N}=2 \mathrm{SYM})$, given by: $W \sim e^{-V}+e^{2 i \pi \tau} e^{V}\left(\tau \equiv \frac{4 \pi i}{g^{2}}+\frac{\theta}{2 \pi}\right)$, where the complex field $V$, formed from the Wilson line for the gauge field along the circle and the scalar dual to the three-dimensional gauge field, parametrize an $\mathcal{N}=2$ Kähler moduli space $\frac{T^{2}}{S^{1}}($ See $[24])$.

The spirit of the paper is similar to the work of, e.g., Sonnenschein et al, in the late nineties - [22] - on seeing whether or not the classical Wilson loop in an $A d S_{5} \times S^{5}$ background, received quantum corrections. In these papers, the authors provide examples of models where the authors explicitly check whether or not one gets a cancelation between the bosonic and the fermionic determinants implying whether or not the classical result for the Wilson loop, receives quantum corrections.

To the best of our knowledge, a spectral/heat kernel asymptotics analysis (based largely on the results in mathematics of Branson, Gilkey and Kirsten) for membrane instantons obtained from a supersymmetric three-fold with boundary, embedded in a $G_{2}$-manifold, has never been worked out, and all the formulae used in this paper are extremely useful not only in the context of membrane instanton superpotential but also quantum corrections to Wilson loops/surfaces.

\section{Acknowledgements}

One of us(AM) would like to thank K.Ray, P.Ramadevi, A.Srivastava and specially A.P.Balachandran for useful correspondences.

\section{References}

[1] A. Volovich , Domain walls in MQCD and Monge-Ampere equation, Phys. Rev. D 59 , 065005 (1999) [arXiv:hep-th/9801166].

[2] E. Witten , Branes and the dynamics of QCD, Nucl. Phys. B 507 , 658 (1997) [arXiv:hep-th/9706109].

[3] D.D.Joyce , Compact Riemannian 7-Manifolds with Holonomy $G_{2}$, I , Jour. Diff. Geom. 43 (1996) , 291; Compact Riemannian 7-Manifolds with Holonomy $G_{2}$, II; Jour. Diff. Geom. 43 (1996) 329; Compact 8-Manifolds with Holonomy Spin(7), Inv. Math. 123 (1996) 507. 
[4] M.Cvetic , G.W.Gibbons , H. Lu and C.N. Pope , New Complete non-compact Spin(7) Manifolds , Nucl.Phys.B620:29-54, 2002 [arXiv:hep-th/0103155]; Supersymmetric M3-branes and $G_{2}$ Manifolds, Nucl.Phys.B620:3-28 , 2002 [arXiv:hep-th/0106026]; Resolved Branes and M-Theory on Special Holonomy Spaces, Strings 2001 [arXiv:hep-th/0106177]; A. Brandhuber , J.Gomis S.S.Gubser and S.Gukov , Gauge Theory at large $N$ and new $G_{2}$ Holonomy metrics, Nucl.Phys.B611:179-204, 2001 [arXiv:hepth/0106034].

[5] A. Misra, On the exact evaluation of the membrane instanton superpotential in M-theory on G(2)holonomy manifold, JHEP 0210 , 056 (2002) [arXiv:hep-th/0205293].

[6] R. Gopakumar and C. Vafa, M-theory and topological strings. I, hep-th/9809187.

[7] V.Periwal, Topological Closed-String Interpretation of Chern-Simons Theory, Phys.Rev.Lett. 71 (1993), 1295.

[8] G. Curio, Superpotentials for M-theory on a G(2) holonomy manifold and triality symmetry, JHEP 0303, 024 (2003) [arXiv:hep-th/0212211]; G. Curio, Superpotential of the M-theory conifold and type IIA string theory, Int. J. Mod. Phys. A 19, 521 (2004) [arXiv:hep-th/0212233].

[9] D. Joyce, On counting special Lagrangian homology 3-spheres, Contemp. Math. 314, 125 (2002) [arXiv:hep-th/9907013].

[10] S. Kachru, S. Katz, A. E. Lawrence and J. McGreevy, Open string instantons and superpotentials, Phys. Rev. D 62, 026001 (2000) [arXiv:hep-th/9912151].

[11] M. Aganagic, A. Klemm and C. Vafa, Disk instantons, mirror symmetry and the duality web, Z. Naturforsch. A 57, 1 (2002) [arXiv:hep-th/0105045].

[12] McKean and I.Singer, J.Diff. Geometry, 1, 43 (1967).

[13] E. Lima, B. Ovrut, J. Park and R. Reinbacher , Non-perturbative superpotential from membrane instantons in heterotic M-theory, Nucl. Phys. B 614, 117 (2001) [hep-th/0101049].

[14] J. A. Harvey and G. W. Moore, Superpotentials and membrane instantons, arXiv:hep-th/9907026.

[15] P.Gilkey, Invariance theory, the heat equation and the Atiyah-Singer Index theorems, 2nd ed (1995) , CRC press; P.Gilkey and T.Branson, Residues of the eta function for an operator of Dirac Type, Journal of Functional Analysis , 108 \#1 (1992) , 47-87.

[16] D. Joyce, Compact Manifolds with Special Holonomy, Oxford University Press, 2000.

[17] E. Witten, On flux quantization in M-theory and the effective action, J. Geom. Phys. 22, 1 (1997) [arXiv:hep-th/9609122].

[18] K. Becker, M. Becker and A. Strominger, Five-branes, membranes and nonperturbative string theory , Nucl. Phys. B 456 , 130 (1995) [arXiv:hep-th/9507158].

[19] G. Cognola, E. Elizalde and S. Zerbini , Dirac functional determinants in terms of the eta invariant and the noncommutative residue, hep-th/9910038. 
[20] S. Deser, L. Griguolo and D. Seminara, Effective QED actions: Representations, gauge invariance, anomalies, and masss expasions, Phys. Rev. D. 57 (1998) 7444 [hep-th/9712066]

[21] V. S. Kaplunovsky, J. Sonnenschein and S. Yankielowicz, Domain walls in supersymmetric Yang-Mills theories, Nucl. Phys. B 552 (1999) 209 [arXiv:hep-th/9811195].

[22] J.Sonnenschein, Wilson loops from supergravity and string theory, J.Sonnenschein, Class. Quant. Grav., 17, 1257 (2000) (hep-th/9910089); Y. Kinar, E. Schreiber, J. Sonnenschein and N. Weiss, Quantum fluctuations of Wilson loops from string models, Nucl.phys.B583:76-104, 2000 (hep-th/9911123).

[23] G. W. Moore, G. Peradze and N. Saulina, Instabilities in heterotic M-theory induced by open membrane instantons, Nucl. Phys. B 607, 117 (2001) [arXiv:hep-th/0012104].

[24] I. Affleck, J. A. Harvey and E. Witten, Instantons And (Super)Symmetry Breaking In (2+1)Dimensions, Nucl. Phys. B 206, 413 (1982); A. Ritz, M. Shifman and A. Vainshtein, Counting Domain Walls In $N=1$ Super Yang-Mills, Phys. Rev. D 66, 065015 (2002) [arXiv:hep-th/0205083] and references therein. 